\section{RCSEd launches app and brand refresh}

The Royal College of Surgeons of Edinburgh (RCSEd) has become the first surgical college in the UK to offer an app to its members in a bid to transform the way training and educational materials are accessed and shared.

The app will be made available to the RCSEd's 29,000 members around the world, giving them access to exclusive content and information on the latest developments in the surgical field.

The launch coincides with the unveiling of the College's new branding, which features Edinburgh Castle, to reflect its long history, and a unicorn, known for its association with healing. The unicorn was also a favourite heraldic animal of James IV, who gave the College its Royal Charter in 1505, with the date also featuring on the logo.

Members will be able to use the app to join interactive webinars with worldleading surgeons, as well as to access hundreds of $3 \mathrm{D}$ videos of dissected human specimens via the Acland Anatomy service.

Library services and transcripts can also be requested from the College Archive and Libraries team through the app, to aid with revision and studying.

The full schedule of College courses, exams and events are now also accessible via an in-app calendar, enabling members to click, save and secure their spot for development opportunities.

Professor Michael Griffin OBE, President of the RCSEd, said: 'The impact of COVID-19 has really demonstrated the importance of being able to teach and learn remotely, and this app will allow us to do even more of this, connecting surgeons like never before and allowing them to share vital information and training.

'In addition to the app, we're also very happy to unveil our new logo, which I believe reflects our friendly, forwardthinking outlook as a College, while also acknowledging our roots as one of the oldest surgical Colleges in the world.'

The app has been developed in conjunction with XDesign.

\section{Updated infection prevention and control guidance published}

Public Health England has published COVID-19: infection prevention and control dental appendix, ${ }^{1}$ which provides updated guidance for dental settings throughout the UK on how to deliver treatment safely during the pandemic.

The guidance is published jointly by Public Health England and the national public health agencies in Scotland, Wales and Northern Ireland, and sets out how dental services should provide treatment for patients on low, medium and high risk COVID-19 pathways. It includes recommendations around aerosol-generating procedures (AGPs), personal protective equipment $(\mathrm{PPE})$, ventilation requirements and postAGP downtime.

The guidance incorporates recommendations contained in the SDCEP report. $^{2}$

\section{Reference}

1. Public Health England. COVID-19: infection prevention and control dental appendix. 20 October 2020. Available at: https://assets.publishing.service. gov.uk/government/uploads/system/uploads/ attachment_data/file/928034/COVID-19_Infection prevention_and_control_guidance_Dental_ appendix.pdf (accessed 26 October 2020).

2. Scottish Dental Clinical Effectiveness Programme. Mitigation of Aerosol Generating Procedures in Dentistry - A Rapid Review. 25 September 2020. Available at: https://www.sdcep.org.uk/publishedguidance/covid-19-practice-recovery/rapid-reviewof-agps/ (accessed 12 October 2020).

\section{BDA AGM}

The BDA Northern Counties Branch AGM will be held online on Thursday 3 December 2020 at 19:00. Please ensure you register your interest in attending at www.bda.org/bse in order to receive the link to access the AGM, or email branchsectionevents@bda.org. 